\title{
A utilização de instrumentos globais para a avaliação da resiliência a desastres na saúde
}

\author{
Use of global instruments for evaluating health disaster resilience
}

Larissa Ferentz', Murilo Noli da Fonseca', Eduardo Pinheiro', Carlos Garcias ${ }^{\mathbf{1}}$

DOI: $10.1590 / 0103-11042020 E 208$

\begin{abstract}
RESUMO Com o aumento dos eventos extremos nas cidades, os gestores locais precisam tomar medidas que minimizem os impactos negativos resultantes dos desastres. A área de saúde é uma das que mais possuem interferências, com o crescimento do número de afetados, feridos, mortos, além dos problemas psicológicos e de qualidade de vida após os eventos. O objetivo da presente pesquisa foi analisar a resiliência a desastres na saúde por meio do instrumento global Disaster Resilience Scorecard for Cities. O Scorecard tem sido utilizado por diversos países para avaliar a resiliência de suas cidades quanto a preparação e resposta na ocorrência de eventos extremos. Por meio da sua aplicação, foi possível observar que apenas a utilização dessa ferramenta se mostra insuficiente para avaliar as diversas dimensões da área de saúde. Além disto, a análise dos Planos Diretores Municipais mostrou que ainda existe a falta de integração entre as medidas de saúde em relação às emergências. É necessário desenvolver leis e ferramentas mais eficientes na avaliação e monitoramento da saúde em caso de desastres, a fim de alcançar a resiliência para uma maior qualidade de vida da população.
\end{abstract}

PALAVRAS-CHAVE Saúde pública. Governo local. Gestão de riscos.

\begin{abstract}
As extreme events in cities increase, local managers need to take measures to minimize the negative impacts of disasters. The health field is among those with most interference, with increasing levels of people who are sick, injured, dead, besides the psychological and quality of life problems after the events. The aim of this research was to analyze health disaster resilience through the global Disaster Resilience Scorecard for Cities instrument. The Scorecard has been used by many countries to assess the resilience of their cities to preparedness and response to extreme events. Through its application, it was observed that only the use of this tool is insufficient to evaluate the various dimensions of the health field. In addition, the analysis of municipal master plans showed that there is still a lack of integration between health measures in relation to emergencies. More efficient laws and tools for health disaster assessment and monitoring need to be developed in order to achieve the resilience for a better quality of life for the population.
\end{abstract}

KEYWORDS Public health. Local government. Risk management.

1 Pontifícia Universidade Católica do Paraná (PUCPR) - Curitiba, (PR), Brasil. ferentzengenharia@gmail. com 


\section{Introdução}

A ocorrência de eventos extremos e desastres tem causado diversas perturbações e impactos no progresso político, social e econômico, e no próprio bem-estar físico, mental e social. $\mathrm{Na}$ saúde pública, essas intercorrências podem causar altos níveis de mortalidade, tragédias e sofrimentos pessoais, consequências imediatas e em longo prazo e interrupções nos serviços de saúde ${ }^{\mathbf{1}}$, deixando as comunidades afetadas sem acesso até mesmo aos serviços básicos de saúde coordenados, integrados e contínuos durante a ocorrência de um desastre.

Como forma de minimizar esses efeitos, faz-se necessário compreender o grau em que os problemas são previstos e reconhecidos, assim como a extensão em que os recursos estão disponíveis ${ }^{2}$. O planejamento e o desenvolvimento de estratégias de prevenção, mitigação e preparação, planos específicos, multisetoriais e multidisciplinares, e mecanismos adequados para a coordenação de recursos antes do evento são elementos críticos e necessários à preparação, potencializando uma resposta bem-sucedida.

Entretanto, diversos obstáculos emergem nesse processo. A integração das políticas e práticas de Redução de Risco de Desastres (RRD) na saúde ainda não é algo plenamente reconhecido. Os esforços para integrar a saúde a esses programas são escassos e frequentemente ocultos nos resultados sociais, econômicos e ambientais ou limitados a referências a salvar vidas ${ }^{3}$. Além do mais, problemas de recursos inadequados e coordenação entre autoridades locais, organizações, profissionais de saúde, disciplinas acadêmicas de saúde e comunidades afetadas antes e no momento da ocorrência de um desastre impedem uma RRD eficaz ${ }^{2}$.

$\mathrm{Na}$ África Ocidental, durante o surto de Ebola (2014-2016), por exemplo, a fragilidade dos sistemas de saúde e a falta de recursos para gerenciar o tratamento dos atingidos sobrecarregaram os serviços de saúde. O desastre resultou em cortes no orçamento para os serviços não relacionados com a doença e uma redução no uso de serviços de saúde, devido ao medo de infecção cruzada. Como consequência, estima-se que mais pessoas tenham morrido de parto, malária, Aids e outras doenças ${ }^{4}$.

Baseado nisso, o presente trabalho tem como objetivo responder à seguinte questão-problema: como o Scorecard (Disaster Resilience Scorecard for cities) pode ser utilizado como instrumento de resiliência municipal ao risco de desastres na área da saúde pública? A partir disso, serão apresentados os resultados obtidos da aplicação desse instrumento, do Escritório das Nações Unidas para a Redução de Risco de Desastres (UNISDR), em três municípios paranaenses: União da Vitória, Campo Largo e Primeiro de Maio. Essa autoavaliação possibilita aos governos locais o monitoramento e análise da sua atuação perante a ocorrência de eventos extremos, a fim de avaliar a resiliência municipal a desastres.

\section{Desastres e seus impactos na saúde pública}

Os desastres resultam da ocorrência de uma ameaça que ocorre em determinada área geográfica, nomeadamente com condições ambientais, materiais e sociais vulneráveis, durante um determinado período, culminando em danos humanos, materiais, ambientais e em prejuízos sociais e perdas econômicas, sobrepujando a resiliência dessas áreas. Na saúde pública, as consequências são complexas e dependem do tipo e das características do fenômeno bem como de sua ocorrência e das condições do território e sociedade. Geralmente, acontecem de forma desigual e variada, direta e indiretamente, e incluem efeitos que ocorrem em tempos diferentes ${ }^{5}$ (figura 1$)^{6}$, variando desde um período curto (horas a poucos dias), caracterizado pelo desenvolvimento de lesões, até longo prazo (meses e anos), quando há surtos de doenças ${ }^{7}$. 
Figura 1. Resposta do setor de saúde em diferentes tempos

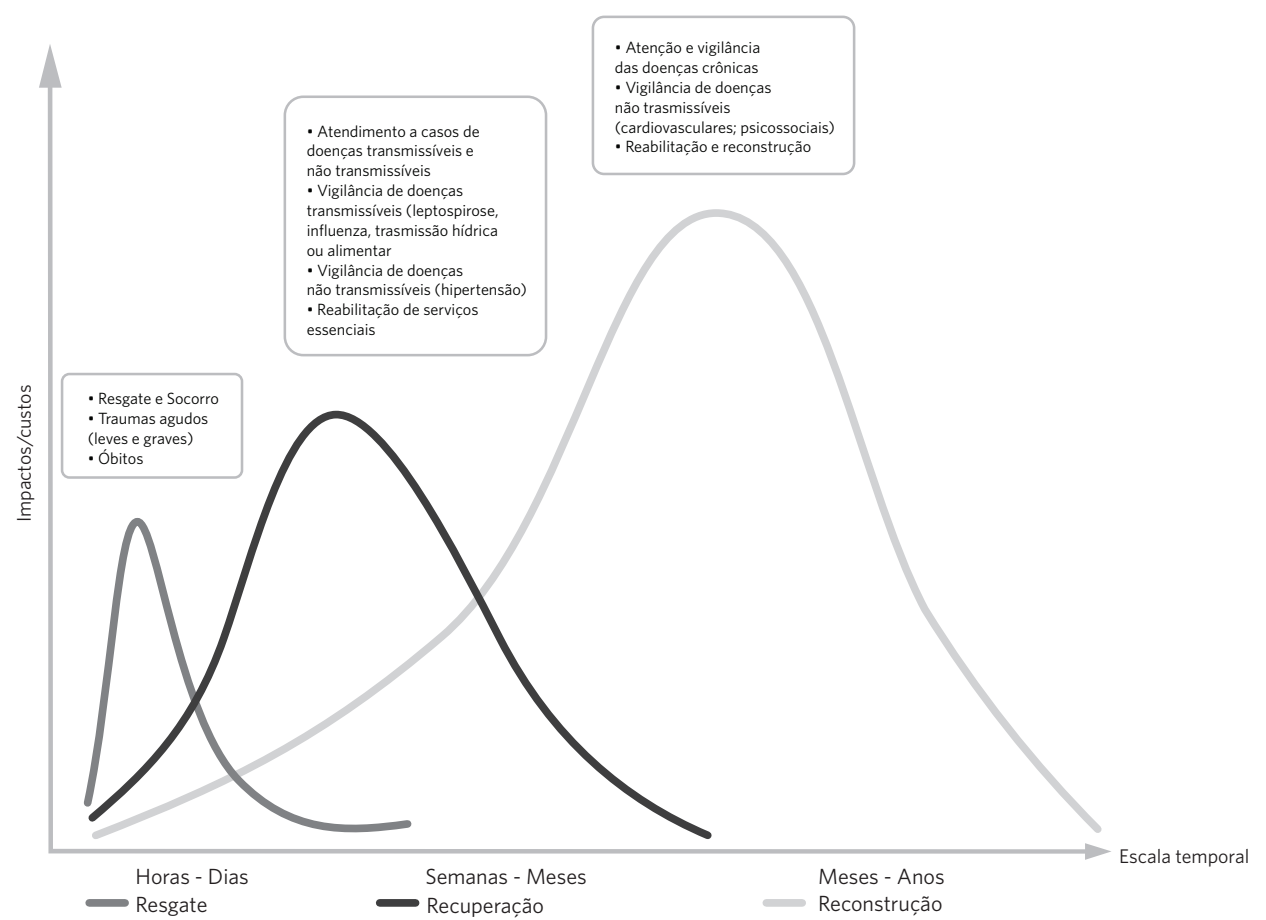

Fonte: Freitas et al.6.

As estratégias de redução dos riscos devem apresentar uma temporalidade maior do que o período em que ocorreram os impactos imediatos após o desastre. Devem se estender ao período de semanas e até meses após a catástrofe, uma vez que contempla ações de vigilância e atenção para as ações de recuperação e construção do sistema urbano e das atividades cotidianas. Não obstante, deve haver uma correlação entre a área setorial saúde e o ciclo que envolve as ações antes, durante e após a ocorrência desses eventos, baseando-se na Política Nacional de Proteção e Defesa Civil, envolvendo as ações de prevenção, mitigação, preparação, resposta a recuperação a eles ${ }^{8}$.

Em um curto prazo (horas a alguns dias), ocorrem mudanças na vida das pessoas, que podem levar a situações de estresse, medo da morte e outros distúrbios da saúde mental ${ }^{9}$. Os indivíduos com doenças crônicas que possuem necessidades médicas regulares podem ter a situação agravada, e muitos correm o risco de óbito quando seus medicamentos não estão disponíveis ou os serviços de saúde são interrompidos. Quando se sabe que cerca de $60 \%$ das mortes por desastres no mundo, nos últimos 50 anos, foram devido a ferimentos causados por esses mesmos eventos ${ }^{10}$, nota-se que o percentual restante tende a possuir relação direta com a interrupção da continuidade e disponibilidade dos serviços de saúde.

Um número significativo de pacientes extravia seus medicamentos durante a evacuação, muitos perdem auxílios médicos, como canetas de insulina, e outros nem sequer têm um registro de suas prescrições quando precisam abandonar com urgência e sem a devida preparação prévia a moradia onde habitam ${ }^{11}$. É nesse período que ocorre a maior parte dos registros de feridos leves e graves e mortalidade6. Por exemplo, após 
a ocorrência de um terremoto, que ocasionou colapso estrutural, houve um considerável incremento no número de visitas hospitalares registradas devido ao número de feridos em um espaço de tempo muito reduzido ${ }^{12}$.

Em um espaço de tempo intermediário, entre dias e semanas, ocorre a emergência de doenças transmissíveis, especialmente a partir da ocorrência de desastres hidrometeorológicos, que facilitam os locais de criação de vetores ${ }^{12}$, como a leptospirose, e a possibilidade de intensificação de doenças não transmissíveis, como a hipertensão. Já entre meses e anos, há o surgimento de doenças não transmissíveis, sobretudo os "transtornos psicossociais e comportamentais, as doenças cardiovasculares, desnutrição e a intensificação de doenças crônicas"6(3647). O transtorno de estresse pós-traumático é a manifestação mais frequente do estresse psicossocial causado por desastres ${ }^{13}$, mas os impactos na saúde mental também incluem sofrimento, ansiedade, consumo excessivo de álcool e outros transtornos psiquiátricos ${ }^{14}$.

Não se pode esquecer também dos impactos na própria infraestrutura (edificações, vacinas, equipamentos, registros) ${ }^{\mathbf{1 5}} \mathrm{e}$ da possibilidade de desassistência, devido à incapacidade, à insuficiência ou à interrupção dos serviços de saúde ${ }^{16}$. Portanto, os desastres agravam os riscos de doenças já existentes na localidade, por isso, a importância da compreensão de como as ações de prevenção em saúde é realizada em consonância com as de prevenção de riscos de desastres em saúde é fundamental.

\section{Gestão municipal de desastres e saúde}

A redução dos impactos dos desastres sobre o sistema urbano em curto e em longo prazo, sobretudo na saúde pública, perpassa pelo desenvolvimento de uma gestão de riscos, desastres e resiliência que vise à implementação de estratégias de redução do grau de exposição às ameaças, diminuição das vulnerabilidades.
Não se pode esquecer também da própria capacitação dos cidadãos para uma melhor preparação e, por conseguinte, enfrentamento perante a ocorrência desses eventos, bem como a participação do sistema de saúde e a colaboração intersetorial e interinstitucional.

O sismo de Kobe, de 1995, que resultou na morte de mais de 5.500 pessoas e $40 \mathrm{mil}$ feridos ${ }^{17}$, por exemplo, provocou alterações no código de construção e estimulou o fortalecimento do sistema de saúde. Essas mudanças, junto com a criação da Associação Japonesa de Medicina de Desastres, tiveram um papel importante na preparação para emergências do sistema de saúde ${ }^{\mathbf{1 8}}$, de modo que elas ajudaram a reduzir o impacto do terremoto e do tsunami de 2011 e, assim, na resiliência ${ }^{19}$.

No entanto, a saúde pública apresenta diversos obstáculos. Recursos limitados e prioridades concorrentes para a prestação de programas comunitários de proteção e promoção da saúde e as atividades de preparação e prontidão das agências de saúde pública atuam, em grande parte, em segundo plano até a ocorrência de um evento ${ }^{20}$. Na China, por exemplo, aproximadamente $9,3 \%$ dos hospitais secundários não revisam e atualizam regularmente os planos de gerenciamento de desastres ${ }^{21}$. Ainda, muitos planos de resgate de emergência atualmente desenvolvidos por hospitais não são práticos ou têm deficiências em termos de logística ou outros fatores relevantes $^{22}$. Na Região Noroeste do estado do Rio de Janeiro, Salles \& Cavalini ${ }^{23}$ aplicaram o Índice de Segurança Hospitalar, que mede a probabilidade de um estabelecimento de saúde continuar funcionando após a ocorrência de um desastre, em dois hospitais públicos e em um privado. Na avaliação, todos foram classificados como categoria B, indicando que podem acontecer prejuízos às estruturas, equipamentos ou serviços.

Desde então, diversos instrumentos foram desenvolvidos para minimizar os impactos de desastres na saúde pública. O Regulamento Sanitário Internacional (RSI), por exemplo, prevê que as capacidades de resposta da saúde 
pública devem envolver: 1) Detectar eventos que apresentem níveis de doença ou óbito acima dos esperados para aquele dado tempo e local, em todo o território do país; 2) Repassar imediatamente todas as informações essenciais disponíveis ao nível apropriado de resposta de atenção à saúde; 3) Implementar imediatamente medidas preliminares de controle ${ }^{\mathbf{2 4}}$. Já o Marco de Sendai para a Redução de Risco de Desastres 2015-2030, que tem uma ênfase maior em saúde do que o seu predecessor, o Marco de Hyogo, apresenta mais de 30 referências à saúde. Elas fazem alusão principalmente à implementação de abordagens de todos os perigos $^{25}$ e vinculadas a epidemias e pandemias além do RSI de 2005. Na Prioridade 3, no nível nacional e local, por exemplo, deve-se

Aumentar a resiliência dos sistemas nacionais de saúde, inclusive integrando o gerenciamento de riscos de desastres aos cuidados de saúde primários, secundários e terciários, especialmente no nível local; desenvolver a capacidade dos profissionais de saúde para entender o risco de desastres e aplicar e implementar abordagens de RRD no trabalho em saúde; e promover e aprimorar as capacidades de treinamento no campo da medicina contra desastres; e apoiar e treinar grupos de saúde comunitária em abordagens de RRD em programas de saúde, em colaboração com outros setores, bem como na implementação do Regulamento Sanitário Internacional de 2005 da Organização Mundial da Saúde26(16).
A resiliência emerge nesse contexto como um elemento que possibilita ao município alcançar um desenvolvimento contínuo e sustentável por meio da integração entre todos os setores governamentais e sociais, de modo que não apenas os sistemas sejam resilientes, mas que todos os cidadãos também se tornem ${ }^{\mathbf{2 7}}$. Logo, ela pode ser definida como sendo a habilidade que um sistema urbano possui para sobreviver, rever ou retornar rapidamente às suas atividades cotidianas após a ocorrência de uma perturbação (natural ou antrópica, repentina ou gradual, em diferentes dimensões de impacto e escalas), para se adaptar à mudança e estabelecer mecanismos funcionais e estruturais de prevenção, preparação e resposta ${ }^{\mathbf{2 8}}$. Ou seja, resulta em uma maior preparação, organização, adaptação e recuperação na ocorrência de desastres.

$\mathrm{Na}$ fase de preparação, por exemplo, Khan et al. ${ }^{20(12)}$ propõem uma estrutura conceitual de resiliência para emergências em saúde pública (figura 2). A estrutura identifica 11 elementos essenciais de um sistema de saúde pública resiliente e como os elementos interagem como um sistema adaptativo complexo. Embora seja focado na preparação, a estrutura faz referência a todas as etapas da gestão de risco de desastres e à promoção da capacidade de adaptação para apoiar a resiliência entre as agências de saúde pública. 
Figura 2. Estrutura de resiliência para a preparação para emergências em saúde pública

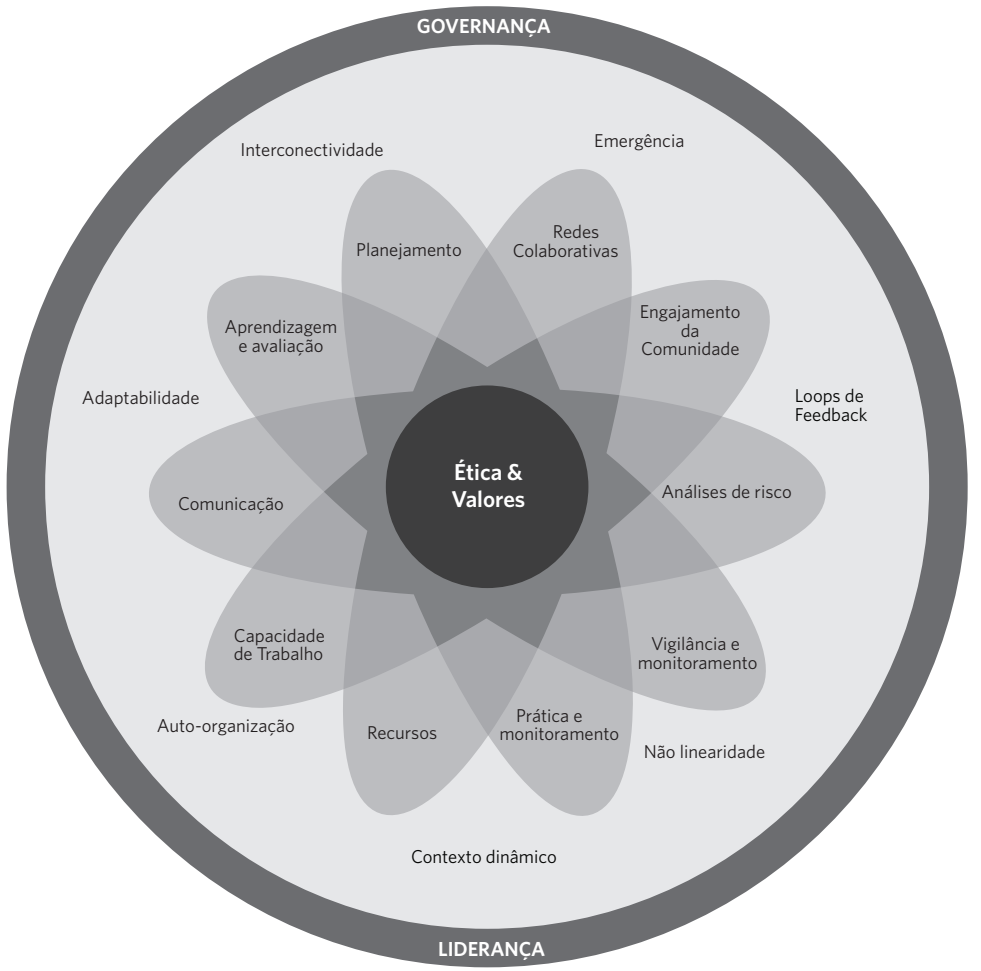

Fonte: Khan et al. ${ }^{\mathbf{2 0}}$

Os elementos são representados como parte do todo, enfatizando o elemento transversal de governança e liderança que envolve os demais. Ele é compreendido como um conjunto de estruturas, parcerias e responsabilidades integradas para dar suporte ao funcionamento coordenado e interoperável do sistema, promovendo clareza e flexibilidade ${ }^{20}$. Em outras palavras, consiste em um meio para facilitar e gerenciar as estratégias de preparação.

\section{Metodologia}

Com a finalidade de compreender como os municípios abordam a gestão de riscos e desastres ao sistema de saúde, empregou-se o instrumento de pesquisa Disaster Resilience Scorecard for Cities. A sua estrutura é baseada nos dez passos essenciais para a construção de cidades resilientes, campanha da UNISDR de 2010, que almeja atender ao Marco de Sendai para a Redução de Risco de Desastres 2015-2030. Segundo a campanha, os dez passos são: 1) organizar-se para a resiliência ante os desastres; 2) identificar, compreender e utilizar cenários de riscos atuais e futuros; 3 ) reforçar a capacidade financeira para a resiliência; 4) promover o desenho resiliente e desenvolvimento urbano; 5) proteger zonas-tampão naturais para melhorar a função da proteção fornecida pelos ecossistemas; 6) fortalecer a capacidade institucional para a resiliência; 7) compreender e fortalecer a capacidade social para a resiliência; 8) aumentar a resiliência da infraestrutura; 9) garantir a eficácia da preparação e resposta eficaz às catástrofes; e 10) acelerar a recuperação e reconstruir melhor depois de qualquer desastre ${ }^{29}$. 
As dimensões 1-3 fazem referência à governança e à capacidade financeira, as 4-8 consideram o planejamento e preparação de desastres, enquanto as 9-10 correspondem à resposta e recuperação pós-desastre. Com essa estrutura, o Scorecard auxilia os municípios a entenderem seus riscos e a conhecerem sua capacidade de mitigação e resposta ante os desastres. Ele também permite análises de curto e longo prazo, considerando os impactos negativos perante as infraestruturas, o meio ambiente e a sociedade ${ }^{30}$.
Dessa forma, utilizaram-se as questões referentes à saúde que estão presentes nos passos oito e nove. Elas fazem referência à segurança estrutural, cuidados de saúde durante desastres, instalações emergenciais (pessoal e primeiros socorros), registros e dados dos pacientes, disponibilidade de atendimento emergencial, incluindo instalações e suprimentos, médicos para necessidades urgentes, capacidade de 'sobretensão' da polícia para apoiar os deveres dos primeiros respondentes e outras necessidades de pessoal e disponibilidade de equipamentos (quadro 1).

Quadro 1. Estruturação do Scorecard para a saúde

\begin{tabular}{|c|c|}
\hline Dimensão & Descrição \\
\hline \multirow{2}{*}{$\begin{array}{l}\text { Segurança estrutural e resiliência a de- } \\
\text { sastres quanto aos cuidados de saúde } \\
\text { e instalações de emergência }\end{array}$} & $\begin{array}{l}\text { Número estimado de leitos em risco x perda de número de dias em cenários } \\
\text { 'mais prováveis' e 'mais severos'. }\end{array}$ \\
\hline & $\begin{array}{l}\text { Número estimado de dias de leito para serviços críticos designados (por } \\
\text { exemplo, quartos de emergência, diálise, terapia intensiva) em risco sob os } \\
\text { cenários 'mais prováveis' e 'mais severos'. }\end{array}$ \\
\hline Registros e dados de pacientes & $\begin{array}{l}\text { \% de pacientes e dados do sistema de saúde e aplicativos associados armaze- } \\
\text { nados e acessíveis em locais improváveis de serem afetados pelo evento. }\end{array}$ \\
\hline $\begin{array}{l}\text { Disponibilidade de atendimentos } \\
\text { emergenciais, incluindo instalações e } \\
\text { suprimentos médicos urgentes para } \\
\text { necessidades agudas }\end{array}$ & Existem recursos de saúde suficientes para lidar com lesões graves. \\
\hline $\begin{array}{l}\text { Capacidade de 'sobretensão' da polí- } \\
\text { cia para apoiar os deveres dos primei- } \\
\text { ros respondentes }\end{array}$ & $\begin{array}{l}\text { Suficiência de apoio ou capacidade profissional para manter a lei e a ordem } \\
\text { em cenários 'mais severos' e 'mais prováveis', além de suportar a carga dos } \\
\text { deveres de primeiro respondente. }\end{array}$ \\
\hline $\begin{array}{l}\text { Definição de outras necessidades de } \\
\text { primeiro respondente e outras neces- } \\
\text { sidades de pessoal e disponibilidade }\end{array}$ & $\begin{array}{l}\text { As necessidades de pessoal são definidas para cenários 'mais prováveis' e } \\
\text { 'mais severos'. }\end{array}$ \\
\hline \multirow{2}{*}{$\begin{array}{l}\text { Definição de necessidades de equipa- } \\
\text { mentos e suprimentos e disponibilida- } \\
\text { de de equipamentos }\end{array}$} & $\begin{array}{l}\text { As necessidades de equipamentos e suprimentos são definidas para os cená- } \\
\text { rios 'mais prováveis' e 'mais severos'. }\end{array}$ \\
\hline & $\begin{array}{l}\text { Desempenho estimado de equipamento disponível por necessidades definidas } \\
\text { - potencialmente de múltiplas fontes. Existem acordos de ajuda mútua com } \\
\text { outras cidades e fontes do setor privado. }\end{array}$ \\
\hline
\end{tabular}

Fonte: United Nations Office for Disaster Risk Reduction ${ }^{30}$.

A pontuação do presente instrumento ocorre em uma escala de 1 a 5 , sendo os resultados de cada dimensão apresentados em forma de percentual. Cabe destacar ainda que, após a aplicação do Scorecard, os dados foram sobrepostos com as legislações vigentes, a fim de validá-los no âmbito legal.

A aplicação do Disaster Resilience Scorecard for Cities se deu em três municípios paranaenses: Campo Largo (Região Metropolitana de 
Curitiba), Primeiro de Maio (Norte Pioneiro) e União da Vitória (Extremo sul). Destaca-se que esses municípios são os pioneiros quanto à aplicação do instrumento de pesquisa no estado do Paraná. Esse fator está relacionado com o interesse dos gestores locais em dar continuidade às etapas da campanha Construindo Cidades Resilientes ${ }^{29}$, a fim de buscar por meios e ações que possam minimizar os riscos de desastres eminentes.

Antes de responder ao instrumento de pesquisa, os municípios tiveram que realizar uma caracterização prévia, identificando o cenário de desastre mais provável (recorrente) e o cenário de desastre mais severo (evento mais grave que já tiveram). Campo Largo identificou como risco de desastre mais provável os alagamentos, e o mais grave, deslizamentos. Primeiro de Maio apresenta vendavais convectivos como os mais frequentes, e epidemia de dengue como o desastre mais grave até hoje enfrentado. União da Vitória possui o mesmo tipo de desastre no cenário mais grave, e nos mais frequentes, as inundações, apresentando alterações apenas em relação às cotas. No cenário provável, o município possui eventos com cotas de 5 metros a 7 metros, e no cenário grave, a cota ultrapassou os 11 metros.

A identificação dos cenários de cada município se faz necessária para responder às questões do Scorecard, considerando a realidade de cada município. Com essa padronização, é possível realizar as comparações, já que cada região deve estar preparada para o enfrentamento de desastres específicos. As questões do instrumento de pesquisa podem ser subdivididas em duas áreas principais, sendo a primeira em relação às infraestruturas de saúde, e a segunda, sobre a ajuda externa de apoio em caso de emergências.

\section{Resultados e discussões}

A dimensão 1, segurança estrutural e resiliência de desastres de cuidados de saúde e instalações de emergência, parte da ideia de que o atendimento dos pacientes, em caso de eventos extremos, poderá continuar a ser fornecido nas instalações existentes ${ }^{30}$. Esse atendimento considera que as instalações são resistentes a desastres ou que existem instalações alternativas designadas para essas ocorrências. Cabe destacar que esse segundo caso não é considerado como o ideal, tendo em vista a necessidade da mudança de deslocamento na rota dos pacientes, assim como as questões de viabilidade estrutural, de equipamentos e de recursos humanos ${ }^{30}$.

Em relação aos casos de disponibilidade de leitos, Campo Largo e Primeiro de Maio marcaram 1 ponto cada, pois ambos os municípios possuem de $10 \%$ a $15 \%$ de leitos perdidos anualmente nos cenários mais prováveis. Em contrapartida, União da Vitória não é atingido nos cenários prováveis, resultando em 4 pontos. Já ao se tratar da disponibilidade de serviços, como os quartos de emergência, Primeiro de Maio obteve apenas 1 ponto, considerando de $5 \%$ a 7,5\% de perdas no cenário mais provável; Campo Largo, 2 pontos, com $2,6 \%$ a $5 \%$ de perdas no cenário mais provável; e União da Vitória, 4 pontos, pois não possui perdas no cenário mais provável. O hospital do município está localizado em uma região alta da cidade, por isso as enchentes não atingem a cota em que ele se encontra.

A dimensão 2, registros e dados de saúde, considera a cobertura das informações do serviço, entre elas: o histórico médico pessoal dos pacientes; os registros dentários (os quais podem ser necessários para a identificação de vítimas); as informações operacionais críticas para as instalações de saúde; e os sistemas de comunicação ${ }^{30}$. Vale ressaltar que a perda de dados precisa ser avaliada em relação ao que existia antes do desastre, por isso é preciso conhecer o que cada município possui na fase de diagnóstico (prevenção).

Os municípios de Campo Largo e Primeiro de Maio não alcançaram pontuação nessa dimensão. Segundo eles, são menos de $80 \%$ os dados que possuem em segurança com aplicativos associados e/ou backups. Destaca-se 
que Primeiro de Maio está com um sistema em fase de implantação, devendo melhorar sua pontuação em um período próximo. Em contrapartida, União da Vitória recebeu pontuação máxima, 5 pontos. O município considera que todos os dados críticos de cuidados com a saúde estão associados a aplicativos, tendo backups rotineiros. Além disso, essas informações estão acessíveis em site remoto, podendo ser acessadas em qualquer lugar, não sendo afetadas pelos eventos extremos que atingem a cidade.

A dimensão 3, disponibilidade de cuidados de saúde de emergência, incluindo instalações e suprimentos médicos urgentes para necessidades agudas, está diretamente ligada à primeira dimensão, no que se refere às perdas estimadas de leitos em dias críticos ${ }^{30}$. Campo Largo e União da Vitória obtiveram 1 ponto cada, pois o tratamento de todas as lesões graves no cenário mais provável, e de $90 \%$ no cenário mais grave, podem ser tratadas dentro de 36 horas.

Em 2014, um dos cenários mais graves de União da Vitória, dois postos de saúde foram atingidos, tendo que encaminhar os pacientes para outras unidades. Eles ficaram inativos por cerca de 30 dias. Em relação a Primeiro de Maio, o município recebeu 3 pontos, pois $o$ atendimento aos pacientes, tanto no cenário mais provável quanto no mais grave, ocorreria dentro de 18 horas. O resumo dessa primeira fase, que considera as infraestruturas de saúde, pode ser observado no gráfico 1 .

Gráfico 1. Resiliência de infraestruturas de saúde (Passo 8)

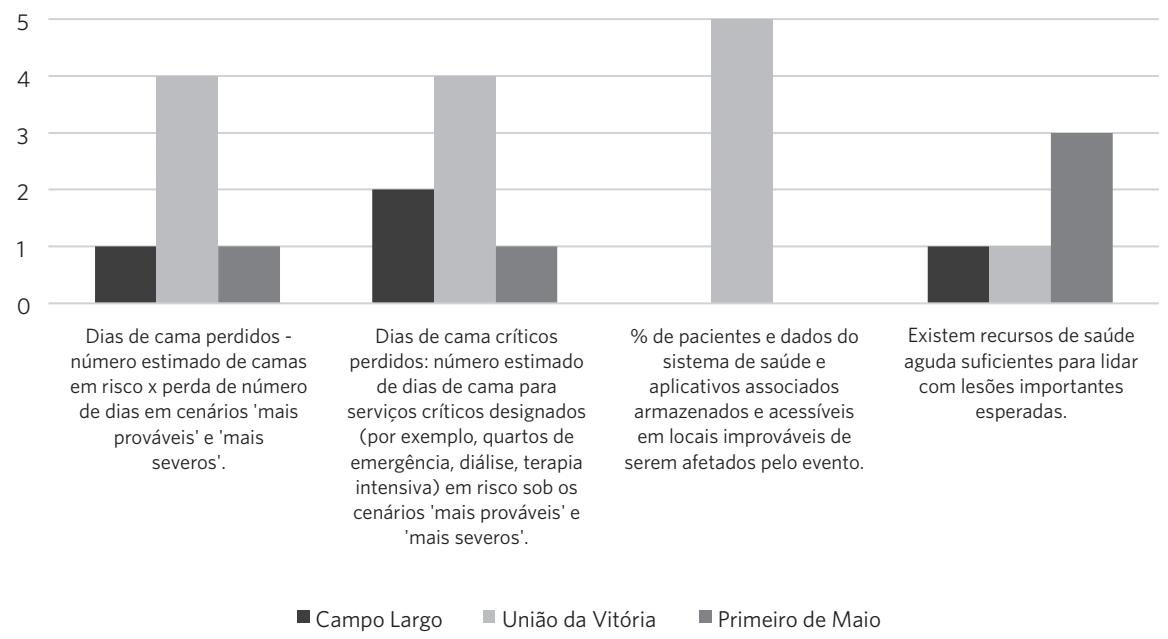

Fonte: Elaboração própria.

Considerando a avaliação das ajudas externas, que complementam as ações dos profissionais de saúde, tem-se a dimensão de número 4, capacidade de 'sobretensão' da polícia para apoiar os deveres dos primeiros respondentes. Além da polícia, essa fase também considera outros organismos-chave, como o Exército ou a Proteção e Defesa Civili30.

União da Vitória foi o único município com pontuação máxima nesse quesito, pois apresenta as necessidades externas definidas, tanto em eventos reais como para treinamentos. 
O apoio recebido é proveniente da Proteção e Defesa Civil, Bombeiros, Exército, Polícia Militar e de diversos voluntários. Primeiro de Maio possui algumas necessidades definidas, mas precisam melhorar em casos de profissões ou áreas específicas da cidade, recebendo 3 pontos. Por fim, Campo Largo alcançou apenas 1 ponto, pois não possui nenhuma necessidade externa previamente definida.

Destaca-se que os municípios possuem essa determinação no escopo de seus Planos Diretores. Entre as principais, estão a disseminação de informações para a população; a mobilização e a capacitação de profissionais e radioamadores; e o estímulo para a participação de organizações não governamentais, associações de classe, voluntários e entidades privadas, para a atuação em caso de eventos extremos.

A dimensão 5, definição de outras necessidades de primeiro respondente e outras necessidades de pessoal e disponibilidade, considera os diferentes padrões nacionais de resposta. Entre eles, estão: ambulâncias, suportes na vizinhança ou em regiões vizinhas, principais pontos de comunicação, pessoas responsáveis pelos setores de água, energia e transporte, entre outros ${ }^{30}$. Parte dessas necessidades podem ser supridas pelas próprias agências governamentais, mas também podem vir de setores privados, com a efetividade dependente de um planejamento contingencial prévio.

União da Vitória novamente teve pontuação máxima, 5 pontos, pois tem todas as necessidades e contatos definidos para o caso de atendimento aos desastres. Primeiro de Maio resultou em 2 pontos, pois a definição dos contatos ainda precisa receber uma melhora expressiva. Ainda não há um serviço de resposta especializado no município. Já Campo Largo recebeu 1 ponto, pois as definições das necessidades não são oficiais. Quando ocorre um evento, o município se comunica com contatos já conhecidos, mas sem ter documentos que liguem esses órgãos à obrigação de auxílio na resposta por exemplo.

Por fim, a última dimensão, número 6 , trata da necessidade e da disponibilidade de equipamentos e suprimentos. Entre eles, incluem-se: veículos de polícia, combate a incêndio e ambulância; helicópteros e aviões; combustível para os equipamentos; escavadeiras e caminhões de detritos; geradores; equipamento de resgate; suprimentos médicos; equipamento manuais (motosserras, guinchos, pás etc.); e sistemas de Tecnologia da Informação (TI) para resposta em emergência local, como dispositivos portáteis ${ }^{30}$. Cabe destacar que esses equipamentos e suprimentos podem ser provenientes tanto de órgãos públicos como de privados.

Em primeiro momento, é realizada a análise das necessidades, que é o conhecimento do que as cidades precisam. União da Vitória foi o único município a atingir a pontuação máxima nesse aspecto, pois apresenta as necessidades de disponibilidade de equipamentos e suprimentos bem definidas. Primeiro de Maio possui algumas necessidades definidas, mas precisam melhorar em algumas áreas específicas da cidade, recebendo 3 pontos. Por fim, Campo Largo alcançou apenas 1 ponto, pois não possui nenhuma necessidade ou plano definido.

Nos Planos Diretores, é possível verificar a previsão de investimentos, planejamento e organização em relação aos equipamentos. Estes estão ligados aos hospitais e postos de saúde pública, centros e serviços especializados, além da rede privada. Como medidas locais, estão a aquisição de novos veículos para suprir as necessidades em cenários normais e emergenciais, além do armazenamento e distribuição de suprimentos e doações durantes os desastres.

Em segundo momento, a análise é realizada em relação à disponibilidade dos equipamentos. Primeiro de Maio recebeu apenas 1 ponto, pois precisa definir mais de $10 \%$ de contatos que possam disponibilizar equipamentos necessários para apoio em situações de desastre ou alternativas para adquiri-los. Campo Largo, com 2 pontos, apresenta-se em uma situação semelhante, precisando definir entre $5 \%$ e $10 \%$. Em contrapartida, União da Vitória possui todos os equipamentos necessários, tendo muito 
bem definidos contatos a quem precisam realizar as solicitações quando necessário, considerando o cenário mais grave. Tendo em vista essa constatação, o município recebeu 5 pontos. O resumo dessa segunda fase, que considera a ajuda externa para uma resposta mais eficaz na área de saúde, pode ser observado no gráfico 2.

Gráfico 2. Resposta eficaz de desastres na saúde (Passo 9)

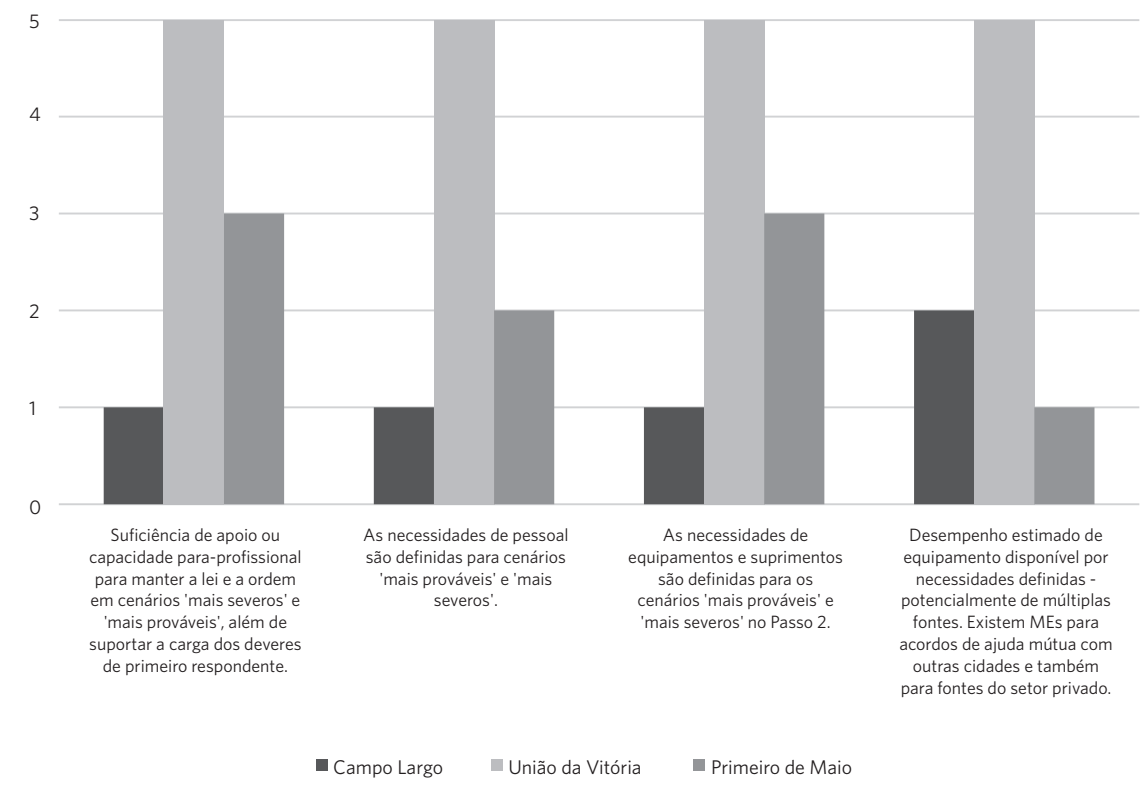

Fonte: Elaboração própria.

Ao ser realizada a análise comparativa baseada nos critérios definidos no rol descritivo da metodologia de autoavaliação Scorecard, percebe-se que há precariedade predominante quanto à relação entre a área setorial saúde e a redução de riscos de desastres. Evidentemente, em muitos municípios e no País, é questionável a própria normalidade operativa da saúde pública, considerando a relação demanda $x$ oferta dos serviços destinados ao atendimento da população.

Entretanto, situações nas quais os desastres se deflagram tendem a piorar e, em não raros casos, a colapsar a infraestrutura existente. A análise dos critérios afeitos à saúde presentes nas etapas avaliadas a partir do Scorecard demonstra que o pensamento e a visão aplicada à essa área, em especial, limitam-se à normalidade, com poucas ressalvas.
União da Vitória é um dos municípios paranaenses que possuem profundas cicatrizes decorrentes de desastres cíclicos ligados à fenômenos de inundação do Rio Iguaçu, dada a sua localização e critérios adotados historicamente para a sua consolidação como cidade, principalmente, extensivo ao município-irmão de Porto União, na outra margem do mesmo rio, no estado de Santa Catarina. Pela recorrência de eventos desastrosos associada à gravidade do conjunto de acontecimentos impostos por essa combinação, que resulta da relação rio e localização da cidade, tem-se a percepção mais nítida quanto à necessidade de inserção de uma visão contingencial no planejamento setorial.

Quando o assunto é desastre - e nenhum deles ocorre sem que haja um risco pretérito 
-, a saúde possui mais que um papel, é detentora de responsabilidades que, provavelmente pela incipiência da inserção temática do risco associado aos demais preceitos da área em tela, sofra ao se ter a aplicação de uma metodologia como a que esses resultados permitem contemplar. Embora a área da saúde ainda seja vista como responsável apenas pelo atendimento quando os desastres já estão ocorrendo, ela tem papel em todas as etapas da gestão de riscos e desastres ${ }^{31,32}$ (quadro 2).

Quadro 2. Processos fundamentais para gestão de riscos e desastres do setor saúde

\begin{tabular}{ll}
\hline Processos & Setor de Saúde \\
\hline Prevenir ris- & A prevenção é o principal processo da redução de riscos e desastres. Requer um enfoque integral com \\
cos futuros & relação aos potenciais danos e à origem de todas ou cada uma das emergências ou desastres possíveis \\
& na realidade do País. \\
& Encontra-se em consonância com a promoção da saúde, com a sustentabilidade ambiental e com a \\
& equidade social. Aponta para a formulação de políticas e ações de saúde sobre os processos de deter- \\
& minação social dos riscos de desastres e de seus impactos sobre a saúde. Demanda articulação inter- \\
& setorial, particularmente com a gestão ambiental e territorial para limitar a ocupação de unidades de \\
& saúde. Envolve a articulação das políticas de saúde com um conjunto de políticas públicas relacionadas \\
& com os determinantes e condicionantes da saúde, como as de geração de emprego e renda, educação, \\
& habitação, meio ambiente, entre outras, que possibilitem que as pessoas vivam em lugares e habitações \\
& saudáveis e seguras.
\end{tabular}

Reduzir A prevenção deve ser realizada simultaneamente com políticas e ações de saúde para minimizar os fariscos exis- $\quad$ tores de riscos já existentes em áreas e populações que se encontram em condições de vulnerabilidade tentes na atualidade, de modo a limitar o impacto adverso das ameaças expressas em situações ou eventos. De modo geral, os desastres potencializam a ampliação e/ou agravamento dos riscos de doenças já existentes nas populações e áreas afetadas. Isso significa que as ações de prevenção em saúde que já são realizadas devem estar integradas com as de prevenção e de surgimento de novas doenças, evitando ou reduzindo a sobreposição de riscos à saúde.

Preparar as A preparação envolve o desenvolvimento de capacidades, instrumentos e mecanismos que permitem respostas antecipadamente assegurar uma resposta adequada e efetiva aos desastres.

A preparação do setor saúde tem como objetivo melhorar a capacidade de resposta na atenção e na vigilância em saúde e evitar que ações inadequadas produzam um segundo desastre (potencializando doenças e agravos já existentes, bem como gerando outros problemas que poderiam ser evitados com medidas preventivas), intensificando os impactos do desastre e comprometendo as ações de recuperação e reconstrução.

Responder A resposta compreende as ações que serão executadas após a ocorrência de um desastre, mas que aos desastres e foram preparadas antes dele, e que têm por objetivo salvar vidas, reduzir o sofrimento humano e diminuir as perdas materiais.

reabilitar as Envolve desde ações de curto e médio prazo, como o cuidado e a atenção para os que sofrem agravos e condições doenças imediatas, até ações de vigilância e monitoramento para implementação de medidas de controle de vida e prevenção de novos fatores de riscos que são gerados por um desastre, como, por exemplo, doenças geradas pelo acesso à água e alimentos contaminados, bem como por vetores e hospedeiros de doenças no pós-desastre. Não só a resposta e a reabilitação são mais efetivas no curto e médio prazo, se baseadas na preparação para respostas, como também evitarão que se prolonguem no tempo se estiverem articuladas com projetos e processos de recuperação e reconstrução das comunidades afetadas.

Recuperar e A recuperação é o processo de reparação da infraestrutura física e do funcionamento definitivo dos reconstruir serviços da comunidade; ao mesmo tempo, envolve promover as mudanças necessárias para a redução comunida- de riscos de desastres futuros.

des Para isso, o setor saúde não só deve proporcionar a continuidade de ações para recuperação e reabilitação da saúde no pós-desastres em consonância com ações de vigilância em saúde como também deve articulá-las com as medidas de reconstrução da comunidade. Recuperar, reabilitar e reconstruir são ações que devem ser realizadas com o objetivo de transformar o desastre em lição e oportunidade para desenvolver e aplicar medidas para reduzir o risco de desastres futuros e tornar as comunidades mais resilientes, promovendo a saúde por meio da sustentabilidade ambiental e da equidade social.

Fonte: Adaptado de Freitas ${ }^{32}$ 
A disparidade em termos de dimensões dos municípios, quando analisamos as suas populações ou até mesmo caso fossem chamados a este debate indicadores econômicos e financeiros de cada municipalidade participante, quantidade de técnicos e sua respectiva qualificação na área da saúde, equipamentos públicos, a comparação entre sua localização e áreas sujeitas a ameaças naturais, valores investidos (orçamentos) da saúde para cada um dos participantes da pesquisa, permitiria ir além enxergando resultados que o Scorecard não consegue chegar, mas que são relevantes.

Apesar de não ser objetivo desta análise, considera-se importante pontuar que se acredita que as condições técnicas e políticas dos municípios, especialmente no que se refere ao planejamento e gestão públicos, prevalecem, inclusive em relação a condições socioeconômicas, na determinação dos resultados. Os aspectos sociais e econômicos possuem restrita interferência direta quanto à escolha dos locais e características das edificações que abrigam os serviços de saúde ou, ainda, quanto ao comportamento e preparação dos gestores nos mais diversos níveis da referida área setorial, os quais podem, com suas ações ou omissões, tomar ou não medidas para prevenir, mitigar ou se preparar para eventuais eventos desastrosos.

A consideração que os municípios deveriam ter em relação à localização em que os funcionários das unidades básicas de saúde e demais instituições públicas e privadas estão com moradia fixa bem ilustra uma das necessidades de preocupação. Embora os municípios devessem ter todo o cuidado para que hospitais não fossem instalados em áreas de risco, ainda existe a problemática daqueles profissionais que trabalham a serviço da população e moram nessas regiões, ficando incapacitados de trabalhar da mesma forma. Quando o setor de saúde do município é prejudicado pela localização da infraestrutura física e, ainda, funcionários habitam em áreas de risco, a resposta durante eventos extremos fica comprometida, pois haverá o aumento da demanda de feridos e a incapacidade local plena de atendimento ${ }^{31}$.

Campo Largo, município com maior população, surpreende ao não apresentar sequer o atendimento aos aspectos avaliados pela metodologia adotada. União da Vitória, a partir da recorrência, demonstra incorporação do risco à área setorial analisada, enquanto Primeiro de Maio - município com menor população, mas localizado numa área do extremo norte do Paraná - obteve índices intermediários. Alcançar um bom resultado não pode significar comodismo, enquanto a precariedade obtida nos índices não precisa estar relacionada com o desânimo, ao contrário, consiste em oportunidade para crescer, implementar e fazer uma incorporação da temática, preferencialmente, tornando-a um caso a ser compartilhado como estímulo aos demais municípios.

Há de ser ressaltado que os municípios não estão sozinhos nessa questão. Estados, o Distrito Federal e a União também possuem competências legais e deveres que lhes chama a desenvolver iniciativas, as quais acabam dando suporte às cidades brasileiras.

Na Secretaria de Vigilância em Saúde (SVS), em 2005, iniciou-se a estruturação na vigilância em saúde ambiental dos desastres naturais (Vigidesastres), a qual passou a integrar a Comissão de Desastres do Ministério da Saúde. Em 2006, foi iniciada a estruturação do Centro de Informações Estratégicas em Vigilância em Saúde (Cievs), com a função de detectar surtos e emergências em saúde pública para disseminar informações para as respostas em saúde. Além disso, houve a criação da Força Nacional do SUS (FN-SUS), em 2011, para atuar em emergências ou desastres em nível nacional. Seguindo essa tendência, em abril de 2012, foi aprovada a Lei $n^{0} 12.608$, que institui a Política Nacional de Proteção e Defesa Civil, que deve se integrar a outras políticas nacionais, entre essas, as de saúde.

Não obstante, em nível global, a saúde urbana também está presente nos conceitos dos Determinantes Sociais da Saúde (DSS), da Organização Mundial da Saúde (OMS), sendo esse um dos pilares para as emergências 
primárias. Esse conceito parte do princípio de que o mal funcionamento de um sistema de saúde pode agravar as situações sociais, econômicas e políticas de uma cidade; apontam-se os três principais fatores determinantes da vulnerabilidade das populações no meio urbano: materiais (habitação, saneamento, transporte, ambienta etc.); psicossociais e comportamentais; e biológicos (genéticos ou não) ${ }^{33}$.

A partir do exposto, é possível verificar que a utilização de apenas esse instrumento global para avaliar a resiliência em saúde nas cidades é muito frágil, pois ele considera apenas questões de atendimento externo para resposta aos eventos e sobre a infraestrutura em que os sistemas de saúde estão inseridos. Um planejamento adequado precisa considerar os diferentes grupos críticos de atendimentos especiais, sendo esses pelo conhecimento prévio das comunidades (crianças, idosos, pessoas com remédios controlados, gestantes e pessoas com necessidades especiais) ou por aqueles mais graves, após os desastres (em choque momentâneo ou com desenvolvimento de problemas psicológicos ao longo dos anos por exemplo).

Além de se apresentar como uma ferramenta incompleta de avaliação, quando sobreposta com as legislações municipais, percebe-se que também falta a integração entre a saúde e as emergências em casos de desastre nos Planos Diretores Municipais. Ademais, a própria saúde deveria elaborar seus planos de contingência - instrumentos de preparação para eventuais situações de resposta a hipóteses de desastres - a partir de premissas que levassem em conta: 1) as tipologias geradas pela própria saúde que pudessem ensejar situações desastrosas; e 2) situações desastrosas específicas que tivessem, como resultado, danos humanos sob a forma de feridos que necessitassem de atendimento urgente e emergencial, demandando a logística e a estrutura de recursos físicos e humanos da saúde. Os municípios não abordaram essas questões nas respostas que pudessem captar tal percepção.

Convém recuperar um aspecto muito relevante mencionado, como exemplificação da assincronia entre a repercussão dos eventos desastrosos na saúde e a necessidade de apuração de danos e prejuízos essenciais para determinar as dimensões que justificariam, em tese, decretações de Situação de Emergência ou de Estado de Calamidade Pública. Os dez dias que o Sistema Nacional de Proteção e Defesa Civil definem como parâmetro são insuficientes para serem captadas algumas doenças e até contaminações posteriores ao prazo para o preenchimento do Formulário de Informação de Desastre (Fide). Talvez uma questão a ser analisada em conjunto entre os órgãos setoriais de saúde nos mais diversos níveis e a defesa civil nacional desse conta da necessidade de efetuar, após monitoramento contínuo das evoluções em diversas áreas, a avaliação multissetorial do impacto do desastre, levando-se em conta os desdobramentos nas mais diversas áreas, e não a pressa em realizar, principalmente, uma contabilização parcial definitiva para o balizamento das ações de recuperação.

É necessário não apenas desenvolver legislações, planos e instrumentos condizentes com as realidades enfrentadas pelos profissionais de saúde, mas também que essas medidas sejam aplicadas, fiscalizadas e melhoradas continuamente, para que, assim, as cidades possuam a tão esperada resiliência em seus sistemas de saúde.

\section{Conclusões}

A Campanha 'Construindo Cidades Resilientes: minha cidade está se preparando!', resulta de uma iniciativa para motivar, dentro de um programa multissetorial, as cidades a melhorarem a incorporação do risco de desastres a todas as áreas setoriais que precisam participar, de forma sistêmica, da questão.

O diagnóstico para conhecer o nível atual e, posteriormente, o monitoramento da resiliência municipal provém da aplicação do Scorecard. Dos dez passos que compõem a campanha, os aspectos intrínsecos à saúde 
foram separados, delimitados e selecionados da aplicação da metodologia aos municípios escolhidos no estado do Paraná, resultando em índices capazes de serem comparados para o conjunto de três cidades: Campo Largo, União da Vitória e Primeiro de Maio.

Os resultados são díspares, apresentando evidências, pelo histórico do município de União da Vitória, que os melhores índices apresentados decorrem da experiência acumulada a partir da recorrência de inundações. Campo Largo destoa por ser a maior cidade em número de habitantes, porém, com dados denotando pouca aplicação de resiliência à área setorial saúde. Primeiro de Maio - com o menor número de habitantes dos municípios avaliados - apresentou números aceitáveis, mas indicando uma grande necessidade de evolução.

Em que pesem as limitações da própria constituição da metodologia adotada, uma vez que, durante as discussões desses resultados, foram apresentadas necessidades que vão além da avaliação da estrutura, mas que se estendem ao nível de preparação dos técnicos e da própria disseminação do conhecimento acerca da relação existente entre saúde, resiliência, risco de desastres, sobretudo iluminando as vulnerabilidades sistêmicas, essa comparação permitiu um diagnóstico preliminar balizador para o planejamento de ações que precisam ser desencadeadas para tornar as cidades mais resilientes. Integrar a questão do risco de desastre à saúde, haver o hábito salutar de planejar a partir de cenários críticos a principal alternativa para reversão dessa realidade constatada.
É inevitável pontuar, como sugestões para futuros estudos, a relação do Scorecard com outras áreas setoriais, ampliando a visão da gestão de riscos e resiliência dos municípios. A saúde, como área setorial, priorizando o nível municipal, precisa delimitar seus próprios indicadores de mensuração da relação com o risco de desastre. Dentro dos dez passos da campanha apresentada como parâmetro para nortear este estudo, verificou-se que o instrumento utilizado possui uma abordagem sintética quanto à temática de saúde, a qual precisa ser desdobrada para ganhar as dimensões que precisa ter, de modo a prepará-la para os eventos desastrosos que certamente afetarão a normalidade dos municípios brasileiros em maior ou menor tempo.

\section{Agradecimentos}

À Coordenação de Aperfeiçoamento de Pessoal de Nível Superior - Brasil (Capes).

\section{Colaboradores}

Ferentz L (0000-0001-5804-0361)* contribuiu para a estruturação do artigo, aplicação dos instrumentos de pesquisa, discussões e revisão do manuscrito. Fonseca MN (0000-0002-0718$3087) *$ contribuiu para estruturação do artigo e discussões. Pinheiro E (0000-0001-54087883)* e Garcias C (0000-0001-7982-5803)* contribuíram igualmente para a coordenação do projeto e discussões. 


\section{Referências}

1. Ting LO ST, Chan EYY, Chan GKW, et al. Health Emergency and Disaster Risk Management (Health-EDRM): Developing the Research Field within the Sendai Framework Paradigm. Inter. J. Disaster Risk Scien. 2017; 8(2):145-149.

2. Khankeh HR, Khorasani-Zavareh D, Johanson E, et al. Disaster Health-Related Challenges and Requirements: A Grounded Theory Study in Iran. Prehospital Disast. Med. 2011; 26(3):151-1588.

3. Murray V. Disaster risk reduction, health, and the post-2015. United Nations landmark agreements. Disast Med Public Health Prepared. 2014; 8(4):283-287.

4. Walker PGT, White MT, Griffin JT, et al. Malaria morbidity and mortality in Ebola-affected countries caused by decreased health-care capacity, and the potential effect of mitigation strategies: A modelling analysis. Lancet. 2015; 15:825-832.

5. Alderman K, Turner LR, Tong S. Floods and Human health: A systematic review. Environment Inter. 2012; 47:37-47.

6. Freitas CM, Silva DRX, Sena ARM, et al. Desastres naturais e saúde: uma análise da situação do Brasil. Ciênc. Saúde Colet. 2014; 19(9):3645-3656.

7. Keim ME. Building human resilience: The role of public health preparedness and response as an adaptation to climate change. American J. Prevent. Med. 2018; 35:508-516.

8. Brasil. Lei Federal n ${ }^{\circ}$ 12608, de 10 de abril de 2012. Institui a Política Nacional de Proteção e Defesa Civil e dá outras providências. Diário Oficial da União. 11 Abr 2012.

9. Williams R, Drury J. Personal and collective psychosocial resilience: Implications for children, young people and their families involved in war and disasters. In: Cook DT, WALL J, editores. Children and armed conflict: Cross-disciplinary investigations. New York: Palgrave Macmillan; 2011. p. 57-75.
10. Centre for Research on the Epidemiology of Disasters. Annual Disaster Statistical Review 2016: The numbers and trends. Belgium: CRED; IRSS; Université catholique de Louvain; 2017.

11. Ochi S, Hodgson S, Landeg O, et al. Disastert-driven evacuation and medication loss: A systematic literature review. PLoS Curr. 2014; 1(18):6.

12. Ahmad J, Ahmad A, Ahmad MM, et al. Mapping displaced populations with reference to social vulnerabilities for post-disaster public health management. Geosp. Health. 2017; 12(576):325-334.

13. Sato H, Techasrivichien T, Omori A, et al. Psychosocial Consequences Among Nurses in the Affected Area of the Great East Japan Earthquake of 2011 and the Fukushima Complex Disaster: A Qualitative Study. Disast. Med. Public Health Prepared. 2018; (28):1-8.

14. Neria Y, Shultz JM. Mental Health Effects of Hurricane Sandy: Characteristics, Potential Aftermath, and Response. JAMA. 2012; 308(24):2571-2572.

15. Pereira CAR, Barata MML. Organização dos serviços urbanos de saúde frente à mudança do clima e ao risco de desastres na América Latina. Saúde debate. 2014; 38(102):624-634.

16. Katarine K, Lemes MMDDL, Andrade M, et al. Os desastres naturais e seus impactos a saúde pública brasileira. Estudos. 2014; 41(2):307-313.

17. Shinfuku N. Disaster mental health: Lessons learned from the Hanshin Awaji earthquake. World Psych. 2002; (1):158-159.

18. Egawa S. Preparedness for low-frequency and high-impact disasters from a medical perspective. The Hyogo framework IRIDeS review report focusing on 2011 Great East Japan Earthquake, 2014; 36:58-67.

19. Aitsi-Selm A, Blanchard K, Al-Khudhairy D, et al. UNISDR STAG 2015 report: Science is use for disaster 
risk reduction [internet]. 2015. [acesso em 2019 set 9]. Disponível em: http://preventionweb.net/go/42848.

20. Khan Y, O'Sullivan T, Brown A, et al. Public health emergency preparedness: a framework to promote resilience. BMC Public Health. 2018; 18(1344):1-16.

21. Gao H, Barbier G, Goolsby R. Harnessing the Crowdsourcing Power of Social Media for Disaster Relief. IEEE Intelligent Systems. 2011; 26(3):10-14.

22. Xieping D, Lufeng S. Current analysis on the disaster medical rescue in foreign countries and China. Clinic. Emerg. 2010; 11(4):207-209.

23. Salles MJP, Cavalini LT. Implementação da "estratégia de hospitais seguros frente a desastres" no Brasil: desafios e evidências empíricas. Hygeia ver. Brasil. Geog. Méd. Saúde. 2012; 14(8):81-90.

24. Organização Mundial da Saúde. Regulamento sanitário internacional: RSI 2005. Brasília, DF: Agência Nacional de Vigilância Sanitária; 2005.

25. Kelman I. Climate change and the Sendai Framework for Disaster Risk Reduction. Inter. J. Disast. Risk Scien. 2015; (6):117-127.

26. United Nations Office for Disaster Risk Reduction. Sendai framework for disaster risk reduction 2015 2030. Geneva: United Nations; 2015.

27. Folke C, Carpenter S, Elmqvist T, et al. Resilience and sustainable development: building adaptive capacity in a world of transformations. Ambio. 2002; 31(5):437-440.
28. Meerow S, Newell JP, Stults MS. Defining urban resilience: A review. Land. Urban Planning. 2016; (147):38-49.

29. Estratégia Internacional das Nações Unidas para a Redução de Desastres. Como Construir Cidades Mais Resilientes: Um Guia para Gestores Públicos Locais. Genebra: Nações Unidas; 2012.

30. United Nations Office for Disaster Risk Reduction. Disaster Resilience Scorecard for Cities: Detailed Level Assessment. Geneva: United Nations; 2017.

31. Silva IVM. Vulnerabilidade institucional do setor saúde a desastres no município de Nova Friburgo. Rio de Janeiro [dissertação]. Rio de Janeiro: Escola Nacional de Saúde Pública Sergio Arouca, Fundação Oswaldo Cruz; 2019. 142 p.

32. Freitas CM. Guia de preparação e respostas do setor saúde aos desastres. Rio de Janeiro: Fiocruz; Secretaria de Vigilância em Saúde; 2018.

33. Ribeiro H. Saúde urbana e sustentabilidade em tempos de globalização. In: Philippi JRA, Collet Bruna G. Gestão Urbana e Sustentabilidade. Barueri: Manole; 2018.

\footnotetext{
Recebido em 30/09/2019

Aprovado em 17/12/2019

Conflito de interesses: inexistente

Suporte financeiro: Coordenação de Aperfeiçoamento de Pessoal

de Nível Superior (Capes). Código Financiamento 001
} 